\title{
Neumomediastino espontáneo en pacientes COVID-19. Serie de casos
}

\author{
Francina V. Bolaños-Morales, ${ }^{1}$ J. Alfredo Santibáñez-Salgado, ${ }^{*}$ Cristóbal Guadarrama-Pérez, ${ }^{2}$ \\ Julio de J. Herrera-Zamora, ${ }^{1}$ Francisco J. Armas-Zárate ${ }^{1}$ y Patricio J. Santillán-Doherty ${ }^{1}$ \\ ${ }^{1}$ Servicio de Cirugía Cardiotorácica; ${ }^{2}$ Departamento de Urgencias. Instituto Nacional de Enfermedades Respiratorias, Ciudad de México, México
}

\section{Resumen}

El neumomediastino espontáneo es la presencia de aire libre en el mediastino sin el antecedente de alguna causa como trauma de tórax. Es una condición benigna autolimitada que se trata en forma conservadora. El diagnóstico clínico se basa en dos síntomas: dolor torácico y disnea; y en un signo en particular: enfisema subcutáneo. Ha sido reportado en pacientes con influenza $A$ (H1N1) y síndrome respiratorio agudo grave; sin embargo, ha sido raramente observado en pacientes con COVID-19. En este trabajo describimos seis pacientes del sexo masculino con COVID-19, con edades entre 27 y 82 años, que presentaron neumomediastino espontáneo y enfisema subcutáneo; ambos se reabsorbieron totalmente con manejo conservador.

PALABRAS CLAVE: Neumomediastino espontáneo. Enfisema subcutáneo. Efecto Macklin. COVID-19.

\section{Spontaneous pneumomediastinum in COVID-19 patients. Case series}

\begin{abstract}
Spontaneous pneumomediastinum is defined as the presence of free air within the mediastinum without an apparent cause such as chest trauma. It is a benign, self-limiting condition that is conservatively treated. Clinical diagnosis is based on two symptoms: chest pain and dyspnea; and on a particular sign: subcutaneous emphysema. It has been reported in patients with influenza $A$ (H1N1) and severe acute respiratory syndrome; however, it has been rarely observed in COVID-19 patients. In this work, we describe six male patients with COVID-19, aged between 27 and 82 years, who presented with spontaneous pneumomediastinum and subcutaneous emphysema; both conditions were completely resorbed with conservative management.
\end{abstract}

KEY WORDS: Spontaneous pneumomediastinum. Subcutaneous emphysema. Macklin effect. COVID-19.

\section{Introducción}

El neumomediastino (NM) espontáneo es la presencia de aire libre dentro del mediastino no precedido por alguna causa como trauma torácico o procedimiento médico o quirúrgico, que causa síntomas como dolor retroesternal, disnea, ronquera y odinofagia Usualmente es una condición benigna y autolimitada, que con frecuencia pasa desapercibida por los médicos. Tiene una incidencia que va de uno entre 8005 a uno entre 42000 ingresos a urgencia. Puede no identificarse con el examen físico, sin embargo, su presencia se sospecha en pacientes con enfisema subcutáneo sin historia de trauma torácico., ${ }^{1,2}$

En esta investigación reportamos seis pacientes masculinos con COVID-19, quienes presentaron NM
Correspondencia:

*J. Alfredo Santibáñez-Salgado

E-mail: alf36@ hotmail.com

DOI: 10.24875/GMM.20000839

0016-3813/৫ 2020 Academia Nacional de Medicina de México, A.C. Publicado por Permanyer. Este es un artículo open access bajo la licencia CC BY-NC-ND (http://creativecommons.org/licenses/by-nc-nd/4.0/).
Fecha de recepción: 20-11-2020

Fecha de aceptación: 10-12-2020

DOI: 10.24875/GMM.20000839
Gac Med Mex. 2021:157:116-120

Disponible en PubMed

www.gacetamedicademexico.com 


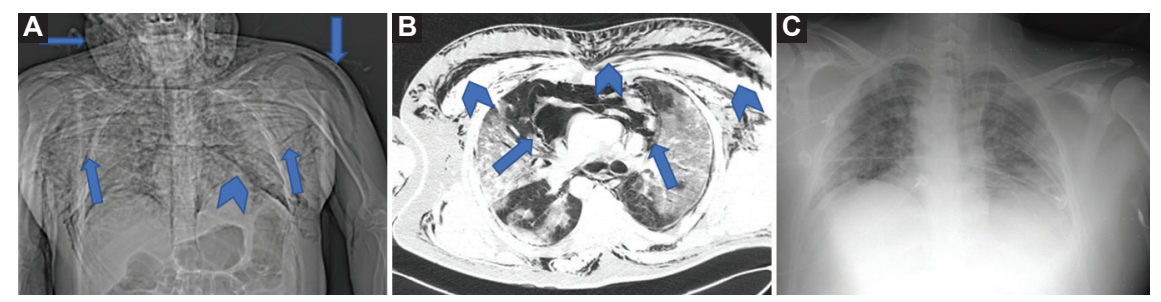

Figura 1. A: Topograma de la tomografía axial computarizada realizada al ingreso en el que se observa enfisema subcutáneo en cara, cuello, tórax y brazos (flechas). B: Tomografía axial computarizada de ingreso, en la que se aprecia neumomediastino y enfisema subcutáneo (cabeza de flecha), con consolidación pulmonar bilateral y áreas de vidrio despulido bilaterales. C: Radiografía de tórax obtenida el séptimo día de hospitalización; no se observa evidencia de neumomediastino ni enfisema subcutáneo.

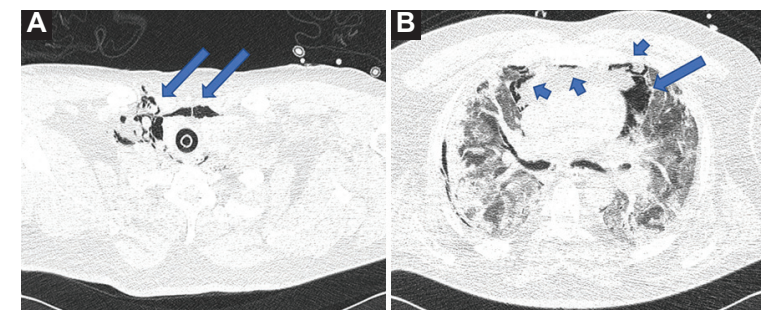

Figura 2. A: Tomografía axial computarizada realizada al ingreso; se aprecia neumomediastino y enfisema subcutáneo (flechas). B: Tomografía axial computarizada realizada al ingreso; se observan áreas de consolidación pulmonar bilateral y áreas de vidrio despulido bilaterales. Neumomedastino (flechas).

espontáneo y enfisema subcutáneo, que se reabsorbió totalmente con manejo conservador.

\section{Métodos}

Se incluyeron pacientes con diagnóstico confirmado de COVID-19, quienes presentaron NM y enfisema subcutáneo. El paciente o la persona legalmente autorizada otorgó el consentimiento informado.

\section{Resultados}

Del 28 de febrero al 30 de septiembre de 2020, 1300 pacientes ingresaron al Instituto Nacional de Enfermedades Respiratorias, Ciudad de México, con el diagnóstico de COVID-19, confirmado con PCR de exudado faríngeo o nasal. Seis pacientes masculinos con edades entre 27 y 82 años presentaron NM espontáneo y enfisema subcutáneo. Cuatro pacientes presentaron NM espontáneo y enfisema subcutáneo antes de su llegada al servicio de urgencias y dos, 24 horas después de haber sido intubados para apoyo mecánico ventilatorio. Un paciente llegó al Departamento de Urgencias con enfisema subcutáneo en cabeza, cuello, tórax y ambos brazos, así como NM diseminado (Figura 1); fue intubado a su ingreso debido a la severidad de la insuficiencia respiratoria. Cinco pacientes presentaron enfisema subcutáneo y NM leve. En las tomografías axiales computarizadas de todos los pacientes se observaron imágenes de vidrio despulido y áreas de consolidación en ambos campos pulmonares (Figuras 1, 2 y 3).

Todos los pacientes presentaron malestar general, dolor torácico, odinofagia y accesos de tos; cinco, disnea severa y baja saturación de oxígeno a pesar del oxígeno suplementario no invasivo. Un paciente con síntomas respiratorios muy leves fue dado de alta a la semana de su ingreso, en buenas condiciones. En todos, el NM espontáneo y el enfisema subcutáneo fueron manejados conservadoramente, con reabsorción total de ambos entre los días 7 y 12 de estancia intrahospitalaria (Figuras 1 y 3). Tres pacientes fueron dados de alta en buenas condiciones de salud y tres fallecieron a causa de complicaciones derivadas de COVID-19. Las características demográficas, manejo y resultado se resumen en las Tablas 1 y 2 .

\section{Discusión}

El neumomediastino espontáneo es el resultado de una variación de presión entre el alveolo y el intersticio pulmonar que deriva en la ruptura alveolar., ${ }^{1,2}$ Dos mecanismos pueden crear este gradiente de presión: el primero es el aumento de la presión intraalveolar (maniobra de Valsalva intencional), descrito como factor precipitante; el segundo es la disminución de la presión en el espacio intersticial perialveolar, que se observa durante un esfuerzo respiratorio intenso o una disminución rápida de la presión atmosférica, los cuales han sido descritos como factores predisponentes. ${ }^{3,4}$ Una vez que sucede la ruptura alveolar, el aire es expulsado centrípetamente a través del intersticio 


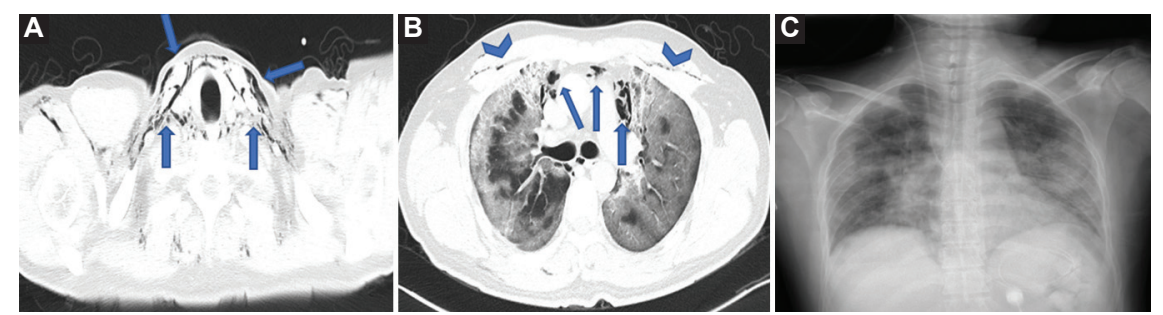

Figura 3. A: Tomografía axial computarizada de ingreso; se observa neumomediastino y enfisema subcutáneo en región cervical (flechas). B: Tomografía axial computarizada de ingreso; se aprecian áreas de consolidación pulmonar bilateral y áreas de vidrio despulido bilaterales, indicativas de neumomediastino (flechas) y enfisema subcutáneo en la región torácica anterior (cabeza de flecha). C: Radiografía de tórax realizada el día 11 de hospitalización; no hay evidencia de neumomediastino ni de enfisema subcutáneo.

Tabla 1. Datos demográficos y evolución clínica

\begin{tabular}{|c|c|c|c|c|c|c|}
\hline Variable & Paciente 1 & Paciente 2 & Paciente 3 & Paciente 4 & Paciente 5 & Paciente 6 \\
\hline Edad (años) & 37 & 47 & 27 & 55 & 38 & 82 \\
\hline Sexo & Masculino & Masculino & Masculino & Masculino & Masculino & Masculino \\
\hline Comorbilidad & Obesidad & Obesidad & No & $\mathrm{ECl}$ & No & Obesidad \\
\hline $\begin{array}{l}\text { Tiempo con síntomas de COVID-19 } \\
\text { antes del ingreso hospitalario }\end{array}$ & 20 días & 10 días & 11 días & 11 días & 7 días & 21 días \\
\hline Apoyo ventilatorio & VMI & VMI & VMI & VMI & No & VMI \\
\hline $\operatorname{PEEP}\left(\mathrm{cm} \mathrm{H}_{2} \mathrm{O}\right)$ & 6 & 8 & 6 & 8 & No & 6 \\
\hline Neumomediastino espontáneo & $\begin{array}{l}\text { Sí (antes de } \\
\text { la VMI) }\end{array}$ & $\begin{array}{l}\text { Sí (antes de } \\
\text { la VMI) }\end{array}$ & $\begin{array}{l}\text { Sí (antes de } \\
\text { la VMI) }\end{array}$ & $\begin{array}{l}\text { Sí ( } 24 \text { horas } \\
\text { después de la VMI) }\end{array}$ & Sí & $\begin{array}{l}\text { Sí (24 horas } \\
\text { después de la VMI) }\end{array}$ \\
\hline Enfisema subcutáneo & $\begin{array}{l}\text { Sí (antes de } \\
\text { la VMI) }\end{array}$ & $\begin{array}{l}\text { Sí (antes de } \\
\text { la VMI) }\end{array}$ & $\begin{array}{l}\text { Sí (antes de } \\
\text { la VMI) }\end{array}$ & $\begin{array}{l}\text { Sí ( } 24 \text { horas } \\
\text { después de la VMl }\end{array}$ & Sí & $\begin{array}{l}\text { Sí (24 horas } \\
\text { después de la VMI }\end{array}$ \\
\hline Neumotórax & No & No & No & No & No & No \\
\hline Tratamiento del NM espontáneo & Conservador & Conservador & Conservador & Conservador & Conservador & Conservador \\
\hline Resolución del NM espontáneo & Día 7 IH & Día $101 \mathrm{H}$ & Día 11 IH & Día $10 \mathrm{IH}$ & Día 7 IH & Día 12 IH \\
\hline Resultado & $\begin{array}{l}\text { Alta el día } \\
27 \| H\end{array}$ & $\begin{array}{l}\text { Falleció el } \\
\text { día } 14 \mathrm{IH}\end{array}$ & $\begin{array}{l}\text { Alta el día } \\
25 \mathrm{H}\end{array}$ & $\begin{array}{l}\text { Falleció el día } \\
22 \| H\end{array}$ & $\begin{array}{l}\text { Alta el día } \\
7 \Vdash H\end{array}$ & $\begin{array}{l}\text { Falleció el día } \\
27 \| H\end{array}$ \\
\hline
\end{tabular}

$\mathrm{PEEP}=$ presión positiva al final de la expiración, $\mathrm{VMI}=$ ventilación mecánica invasiva, $\mathrm{IH}=$ intrahospitalario, $\mathrm{ECl}=$ enfermedad cardiaca isquémica, $\mathrm{NM}=$ neumomediastino.

pulmonar a lo largo de las vainas broncoalveolares hacia el hilio pulmonar y el mediastino, y puede continuar hacia los planos subcutáneos cervicales y la pleura. Este mecanismo fisiopatológico se conoce como efecto Macklin. ${ }^{1-4}$ La presencia de al menos un factor precipitante como acceso de tos, emesis y ejercicio vigoroso se observó en 21 a $88.9 \%$ de los pacientes y al menos un factor predisponente como fumar, asma, infección respiratoria y enfermedad pulmonar intersticial estuvo presente en $22 \%$ de los pacientes con NM espontáneo. ${ }^{3,4}$

El alveolo es propenso a romperse en pacientes con neumonía viral severa debido a que el virus daña la membrana alveolar ya que infecta a los neumocitos tipos I y II. La mayoría de los pacientes con neumonía viral tiene al menos el factor precipitante de la tos, la cual puede ocasionar la ruptura alveolar y el desarroIlo de NM. ${ }^{4-6}$

Los pacientes descritos presentaban tos seca, dolor torácico, mialgia y malestar general; cuatro, fiebre, crepitaciones subcutáneas sin antecedentes de trauma torácica ni manipulación de la vía aérea o el esófago. Dos pacientes desarrollaron NM y enfisema subcutáneo 24 horas después de haber sido intubados para apoyo mecánico ventilatorio invasivo. Establecimos la hipótesis de que el NM no estaba relacionado con la 
Tabla 2. Signos, síntomas y gases sanguíneos de los pacientes a su llegada a urgencias

\begin{tabular}{|c|c|c|c|c|c|c|}
\hline Parámetro & Paciente 1 & Paciente 2 & Paciente 3 & Paciente 4 & Paciente 5 & Paciente 6 \\
\hline Tos & Accesos severos & Accesos severos & Accesos leves & Accesos leves & Accesos leves & Accesos severos \\
\hline Dolor torácico & Sí & Sí & Sí & Sí & Sí & Sí \\
\hline Odinofagia & Sí & Sí & Sí & Sí & Sí & Sí \\
\hline Malestar general & Sí & Sí & Sí & Sí & Sí & Sí \\
\hline Temperatura $\left({ }^{\circ} \mathrm{C}\right)$ & 36.5 & 39.6 & 37 & 38 & 37.1 & 38 \\
\hline Disnea & Sí & Sí & Sí & Sí & No & Sí \\
\hline $\begin{array}{l}\text { Frecuencia cardiaca } \\
\text { (× minuto) }\end{array}$ & 110 & 120 & 106 & 104 & 90 & 100 \\
\hline $\begin{array}{l}\text { Frecuencia respiratoria } \\
\text { (× minuto) }\end{array}$ & 34 & 30 & 40 & 30 & 20 & 40 \\
\hline Saturación de $\mathrm{O}_{2}$ & $\begin{array}{c}43 \% \text { con } 15 \mathrm{~L} / \mathrm{min} \\
\text { puntas nasales }\end{array}$ & $\begin{array}{c}65 \% \text { con } 15 \mathrm{~L} / \mathrm{min} \\
\text { puntas nasales }\end{array}$ & $\begin{array}{c}86 \% \text { con } 10 \mathrm{~L} / \mathrm{min} \\
\text { puntas nasales }\end{array}$ & $\begin{array}{c}56 \% \text { con } 15 \mathrm{~L} / \mathrm{min} \\
\text { puntas nasales }\end{array}$ & $\begin{array}{l}96 \% \text { aire } \\
\text { ambiente }\end{array}$ & $\begin{array}{c}80 \% \text { con } 15 \mathrm{~L} / \mathrm{min} \\
\text { puntas nasales }\end{array}$ \\
\hline $\mathrm{pH}$ & 7.19 & 7.42 & 7.45 & 7.15 & 7.46 & 7.17 \\
\hline $\mathrm{pO}_{2}(\mathrm{~mm} \mathrm{Hg})$ & 46 & 24 & 58.2 & 65.3 & 93.6 & 82.9 \\
\hline $\mathrm{pCO}_{2}(\mathrm{~mm} \mathrm{Hg})$ & 68.7 & 24 & 30.6 & 69.1 & 30 & 25.8 \\
\hline $\mathrm{HCO}_{2}(\mathrm{mmol} / \mathrm{L})$ & 25 & 16.2 & 21.3 & 18.8 & 27.3 & 9.1 \\
\hline
\end{tabular}

ventilación mecánica invasiva. Se ha reportado que la ruptura alveolar no está relacionada con el uso de presión positiva al final de la expiración en los pacientes con síndrome respiratorio agudo severo, sino con apoyo mecánico ventilatorio invasivo con presiones meseta mayores de $35 \mathrm{~cm}$ de $\mathrm{H}_{2} \mathrm{O}^{5-8}$ En nuestros pacientes, el apoyo mecánico ventilatorio invasivo no empeoró el NM ni el enfisema subcutáneo.

El enfisema subcutáneo y el antecedente de accesos intensos de tos sugirieron el diagnóstico de NM espontáneo, el cual fue confirmado mediante radiografía y tomografía axial computarizada de tórax. La radiografía de tórax es el estándar de oro para diagnosticar NM espontáneo, sin embargo, la mitad de los casos pueden pasar desapercibidos sin la radiografía lateral de tórax, ${ }^{1,2}$ la cual no puede efectuarse en los pacientes con apoyo mecánico ventilatorio invasivo. La tomografía axial computarizada de tórax es el estudio de imagen más confiable para confirmar el NM espontáneo; sin embargo, el seguimiento se lleva a cabo con radiografías de tórax seriadas debido a la posibilidad de neumotórax, que puede ocasionar compromiso cardiopulmonar y cuyo tratamiento requerirá la instalación de un sistema de drenado o tratamiento quirúrgico si continúa la fuga aérea. ${ }^{9}$
La evolución clínica del NM espontáneo y del enfisema subcutáneo en nuestros pacientes fue buena, con la reabsorción gradual del aire por los tejidos, a pesar de la gravedad de COVID-19. Comúnmente el NM espontáneo es una condición benigna autolimitada, por lo que es suficiente el manejo conservador. ${ }^{9}$

\section{Conclusiones}

Los pacientes con COVID-19 presentan accesos de tos, los cuales pueden causar NM espontáneo y enfisema subcutáneo. EI NM se sospecha en pacientes con enfisema subcutáneo cervical o torácico sin antecedente de trauma torácico. El seguimiento clínico y la evaluación radiológica son suficientes para detectar mejoría o complicaciones cardiorrespiratorias. Los pacientes con NM pueden ser manejados conservadoramente y la presión positiva de la ventilación mecánica invasiva no agravan el NM ni el enfisema subcutáneo.

\section{Conflicto de intereses}

Los autores declaran que no tienen algún conflicto de intereses. 


\section{Financiamiento}

No se recibió financiamiento económico para la realización de este trabajo.

\section{Responsabilidades éticas}

Protección de personas y animales. Los autores declaran que para esta investigación no se realizaron experimentos en seres humanos ni en animales.

Confidencialidad de los datos. Los autores declaran que siguieron los protocolos de su centro de trabajo sobre la publicación de datos de pacientes.

Derecho a la privacidad y consentimiento informado. Los autores obtuvieron el consentimiento informado de los pacientes o sujetos referidos en el artículo.

\section{Bibliografía}

1. Okada M, Adachi H, Shibuya Y, Ishikawa S, Hamabe Y. Diagnosis and treatment of patients with spontaneous pneumomediastinum. Resp Invest. 2014;52:36-40

2. Kim KS, Jeon HW, Moon Y, Kim YD, Ahn MI, Park JK, et al. Clinical experience of spontaneous pneumomediastinum: diagnosis and treatment. J Thorac Dis. 2015;7:1817-1824.

3. Meireles J, Neves S, Castro A, Franca M. Spontaneous pneumomediastinum revisited. Respir Med CME. 2011;4:181-183.

4. Dionísio P, Martins L, Moreira S, Manique A, Macedo R, Caeiro F, Boal L, Bárbara C. Spontaneous pneumomediastinum: experience in 18 patients during the last 12 years. J Bras Pneumol. 2017;43:101-105.

5. Chekkoth SM, Naga SR, Valsala N, Kumar P, Raja RS. Spontaneous pneumomediastinum in $\mathrm{H} 1 \mathrm{~N} 1$ infection: uncommon complication of a common infection. J R Coll Physicians Edinb. 2019;49:298-300.

6. Chu CM, Leung YY, Hui JYH, Hung IFN, Chan VL, Leung WS, et al. Spontaneous pneumomediastinum in patients with severe acute respiratory syndrome. Eur Resp J. 2004;23:802-804.

7. Wang J, Su X, Zhang T, Zheng C. Spontaneous pneumomediastinum: a probable unusual complication of coronavirus disease 2019 (COVID-19) pneumonia. Korean J Radiol. 2020;21:627-628.

8. Boussarsar M, Thierry G, Jaber S, Roudot-Thoraval F, Lemaire $F$, Brochard L. Relationship between ventilatory settings and barotrauma in the acute respiratory distress syndrome. Intensive Care Med. 2002;28:406-413.

9. Kouritas VK, Papagiannopoulos K, Lazaridis G, Baka S, Mpoukovinas I, Karavasilis V, et al. Pneumomediastinum. J Thorac Dis. 2015;7:S44-S49. 